\title{
Altos estructurales costa afuera en la región de Valparaíso, Chile central
}

\author{
Offshore structural highs at Valparaíso region, central Chile \\ Hernán P. Vergara ${ }^{1}$ y René Astudillo ${ }^{1}$ \\ ${ }^{1}$ Facultad de Ciencias del Mar y de Recursos Naturales, Universidad de Valparaíso, Casilla 5080 Reñaca, Viña del Mar, Chile \\ hernan.vergara@uv.cl
}

\begin{abstract}
By means of satellite 3-D altimetry, a $40 \mathrm{~km}$ submarine alignment sited at $32^{\circ}-33^{\circ}$ south latitude on the shelf break in slope, $27 \mathrm{~km}$ offshore Valparaíso region, was detected and described. The alignment consists of three peaks at 200, 733 and $880 \mathrm{~m}$ below sea level bearing $\mathrm{N} 10^{\circ} \mathrm{W}$ and showing 1230,1350 and $1545 \mathrm{~m}$ relief relative to the surface of the upper slope. According to the international standards for undersea features, the names of Los Molles, Horcón and Zapallar are proposed for them in a northward trend. The whole morphological pattern suggests a non confined block displacement of the outer shelf relative to the surface of the upper slope border implying a Holocene subsidence displacement possibly related to the stresses and seismicity generated by the subduction of the Papudo asperity below the middle slope.
\end{abstract}

Key words: Satellite altimetry, submarine morphology, continental margin

\section{Introducción}

Entre los años 2003 y 2007, el Grupo de Geodesia Satelital de Scripps (SIO) de la Universidad de California en San Diego (UCSD) puso a disposición de la comunidad científica internacional la cartografía de alta resolución de los fondos marinos, recopilada por una gran cantidad de cruceros de investigación efectuados en los últimos 30 años, a los cuales añadió la información batimétrica obtenida por altimetría satelital en tres dimensiones (Sandwell \& Smith 1997, 2001, Tapley \& Kim 2001, Sandwell et al. 2006).

En el presente trabajo se aplica esta última parte de la información a la detección, actualización y complementación de la morfología submarina al interior de las 12 millas náuticas de Mar Territorial, costa afuera de Chile central, entre $\operatorname{los} 32^{\circ}$ y $33^{\circ} \mathrm{S}$, habitualmente deficitaria en este tipo de información.

El objetivo del estudio es caracterizar la morfología de altos estructurales aparentemente vinculados a un evento de deformación tectónica del Holoceno.
Resumen.- Mediante altimetría satelital 3-D se detectó y describió un lineamiento submarino de $40 \mathrm{~km}$ ubicado en el quiebre de pendiente plataforma-talud continental, entre los $32^{\circ}$ y $33^{\circ} \mathrm{S}, 27 \mathrm{~km}$ costa afuera de la región de Valparaíso. El alineamiento consiste en tres cumbres a 200, 733 y $880 \mathrm{~m}$ de profundidad orientadas al $\mathrm{N} 10^{\circ} \mathrm{W}$, con relieves de 1230,1350 y $1545 \mathrm{~m}$ respectivamente desde la base a la cima. Se propone designarlos como Los Molles, Horcón y Zapallar en su orientación hacia el norte, de acuerdo a la normativa internacional para los relieves submarinos. El patrón morfológico sugiere un dislocamiento holocénico de un bloque no confinado del borde externo de la plataforma, con relación al borde superficial del talud superior, posiblemente vinculado a mecanismos tensionales y la sismicidad generada por la subducción de la aspereza Papudo bajo el talud medio.

Palabras clave: Altimetría satelital, morfología submarina, margen continental

\section{Material y métodos}

La batimetría se obtuvo de la base de datos de Sandwell \& Smith (2001), del Laboratorio de Geodesia Satelital de SIO, en formato SRTM (Shuttle Radar Topography Mission), que fue procesada y transformada a modelos digitales de elevación (DEM), mediante los cuales se obtuvieron planos en 3-D de realidad virtual con diferentes espaciamientos de isóbatas. El posicionamiento del área se estableció con imágenes Landsat en formato MrSid en proyección UTM 19 referida al datum WGS84.

\section{Marco regional}

La morfología, estructura interna y sismicidad del margen continental frente a la región de Valparaíso ha sido actualizada por diversos autores (Yáñez et al. 2001, Contreras 2007, Thierer et al. 2005), quienes han estudiado las anomalías magnéticas, la evolución del margen continental y las características de sismicidad en el margen de la región de Valparaíso. 
El zócalo de la costa y de la plataforma es un granito leucocrático cuya composición varía desde granodioritas hasta tonalitas gneísicas de grano grueso (Alvarez 1964, Valenzuela et al. 1979). Sobre el zócalo se encuentra la Formación Navidad (Mioceno tardío) constituida por una secuencia sedimentaria fosilífera marina con limolitas, areniscas arcillosas, areniscas de grano fino a grueso, bancos de conglomerados, coquinas y calizas (Tavera 1979, Cecione 1980, Martínez-Pardo 1990, Frassinetti 1993).

El margen continental de la zona central de Chile entre los $32^{\circ}$ y $33^{\circ} \mathrm{S}$ es bastante complejo. Perpendicularmente a la costa ocurre un particular fenómeno morfotectónico; se halla dividido en tres provincias fisiográficas: el talud superior que presenta una tectónica de bloques, una terraza colgante con una potencia importante de sedimentos que alcanza a varios kilómetros y el talud inferior que presenta un prisma de acreción y asperezas bajo él (von Huene et al. 1997, Yáñez et al. 2001).

En el talud superior hay dos cañones submarinos, La Ligua y Montemar (Vergara 1996) que convergen hacia la cuenca Valparaíso, donde desaparecen bajo el relleno sedimentario. La traza zigzagueante de ambos cañones evidencia un control estructural inducido por una tectónica de bloques (Scholl et al. 1970, von Huene et al. 1997).
En el talud continental destaca la morfología de la cuenca Valparaíso, de $62 \mathrm{~km}$ de largo por $45 \mathrm{~km}$ de ancho entre las isóbatas 2.450 y $2.800 \mathrm{~m}$, con una superficie de $2.790 \mathrm{~km}^{2}$. La cuenca está rellena con un prisma sedimentario cuyo espesor fluctúa entre $2 \mathrm{~km}$ en la parte norte y más de $4 \mathrm{~km}$ en el sector sur. Durante el crucero $80 \mathrm{a}$ del R/V Sonne se extrajeron 2 testigos: el No $17748-2$ (32 $2^{\circ} 45.0$ 'S $\left.72^{\circ} 02.0^{\prime} \mathrm{W}\right)$, de $383 \mathrm{~cm}$ de largo, a la profundidad de 2.545 m y el GwB No $3302-1$ (33⒔1'S - 7205.4'W), de $412 \mathrm{~cm}$ de largo, a la profundidad de $1.498 \mathrm{~m}$ (Stoffers et al. 1992). Su litología fue descrita por Vergara (1996) y Stoffers et al. (1992) y muestra que los sedimentos superficiales son fangos biógenos con intercalaciones de arenas bioturbadas que sugieren un transporte turbulento de los estratos inferiores, con gradación normal y velocidades de sedimentación decreciente en función del tiempo geológico.

El talud inferior se caracteriza por una aspereza asociada a la corteza oceánica (monte submarino Papudo) que al subductar disloca el prisma de acreción y el borde continental (Yáñez et al. 2001, figuras 7 y 15), dejando una cicatriz morfológica de $10 \mathrm{~km}$ de ancho en el talud inferior, que se manifiesta en la presencia del Cordón Punta Salinas (Yáñez et al. 2001).

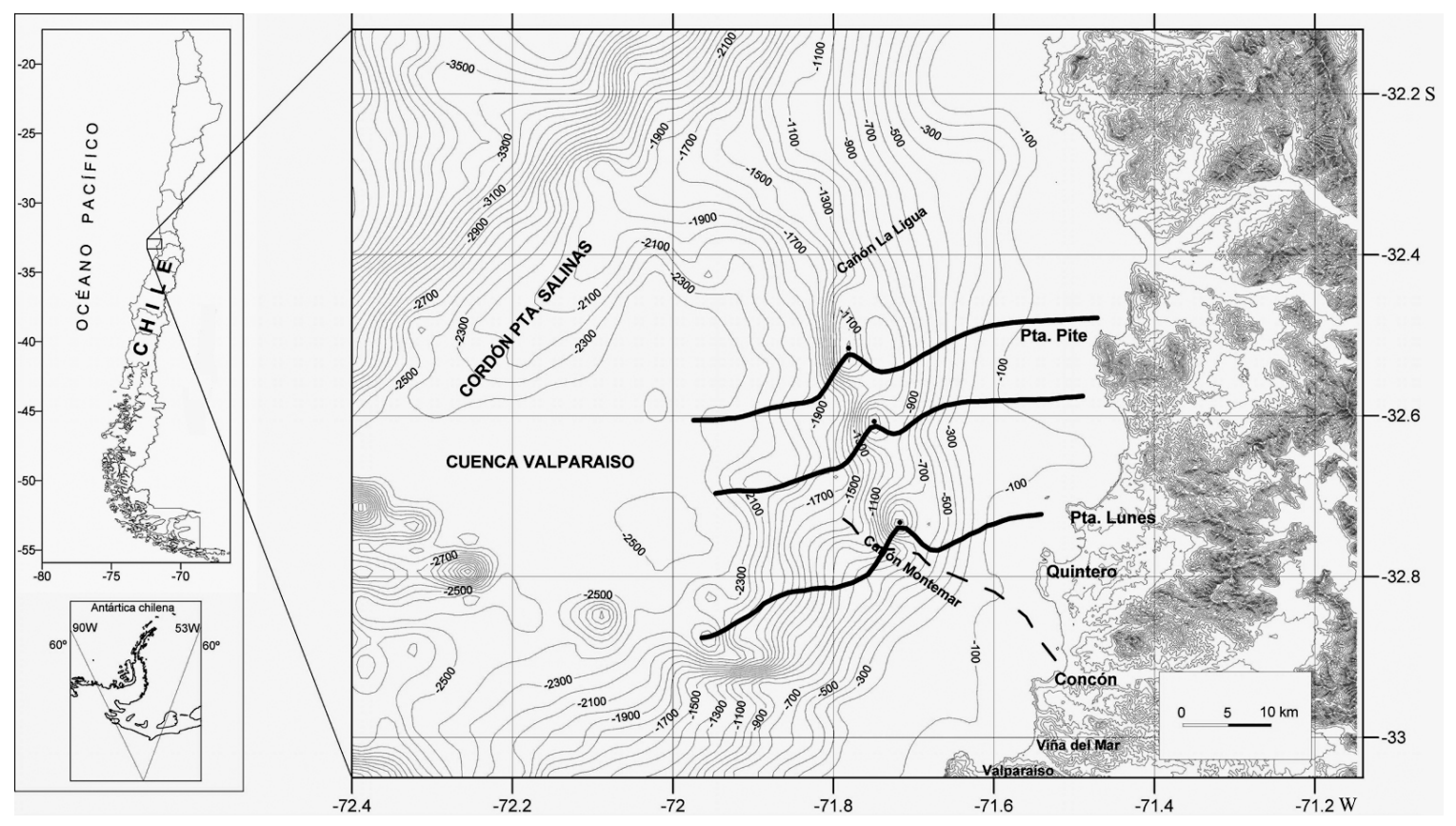

Figura 1

Mapa de ubicación del área de estudio mostrando la topografía costera y batimetría de la plataforma submarina, talud y cuenca de Valparaíso. Los trazos negros representan los perfiles transversales de los altos estructurales y la línea delgada segmentada conecta el Cañón Montemar con la boca del río Aconcagua

Location map with the studied area showing the coastal topography and bathymetry of the shelf, upper slope and Valparaíso Basin. Black lines sketch the profile of the structural highs and the segmented thin line connects the Montemar Canyon head to the Aconcagua River mouth 


\section{Resultados y discusión}

El área de estudio se ubica $27 \mathrm{~km}$ costa afuera de la región de Valparaíso, en el quiebre de pendiente entre la plataforma continental y el talud oceánico, a los $32,6^{\circ} \mathrm{S}$ (Fig. 1). Allí se detecta un bloque de $40 \mathrm{~km}$ de longitud caracterizado por tres cumbres (Fig. 2) escalonadas de orientación $\mathrm{N} 10^{\circ} \mathrm{W}$ a alturas promedio sobre el plano del talud de $1.230,1.350$ y $1.545 \mathrm{~m}$ (Tabla 1$)$. Sus cimas están, de sur a norte, a 200, 733 y $880 \mathrm{~m}$ de profundidad, respectivamente. Formalmente se propone designarlos como Los Molles, Horcón y Zapallar (International Hydrographic Organization 2001).

En el recuadro a) de la Fig. 3 se muestra la morfología del talud superior y se destaca la ubicación de los cañones submarinos La Ligua y Montemar, que por el norte y sur limitan la extensión de un relieve escalonado emplazado entre la plataforma y la cuenca Valparaíso. En el recuadro b) de la misma figura se ilustra detalladamente la morfología del relieve escalonado y la traza del Cañón Montemar.
El aspecto de la morfología generalizada (Fig. 3) sugiere el desplazamiento vertical de un bloque no confinado del borde externo de la plataforma. Su confinamiento se debe a esfuerzos causados por la subducción de la aspereza Papudo bajo el talud que genera fallamientos en el margen, con la consiguiente subsidencia del bloque. Este mecanismo posiblemente lo vincule con la sismicidad del sector epicentral Quintero-Papudo. En base a estos rasgos morfológicos se infiere que la génesis de las cumbres es posterior a la del talud y la plataforma continental, actualmente datada como posterior al último ascenso del nivel del mar, iniciado hace 10.000 años AP y estabilizado a los $33^{\circ} \mathrm{S}$ en los 4.000 años AP (Latorre et al. 2007). Lo anterior es confirmado por el desplazamiento de las isóbatas de la cabecera del cañón Montemar y en la base del alto estructural Los Molles (Figs. 1 y 2), que evidencian que el borde de la plataforma ha sido modificado. Basados en ello, se concluye que el dislocamiento y rotación de un bloque no confinado del talud ocurrió con seguridad durante el Holoceno superior.

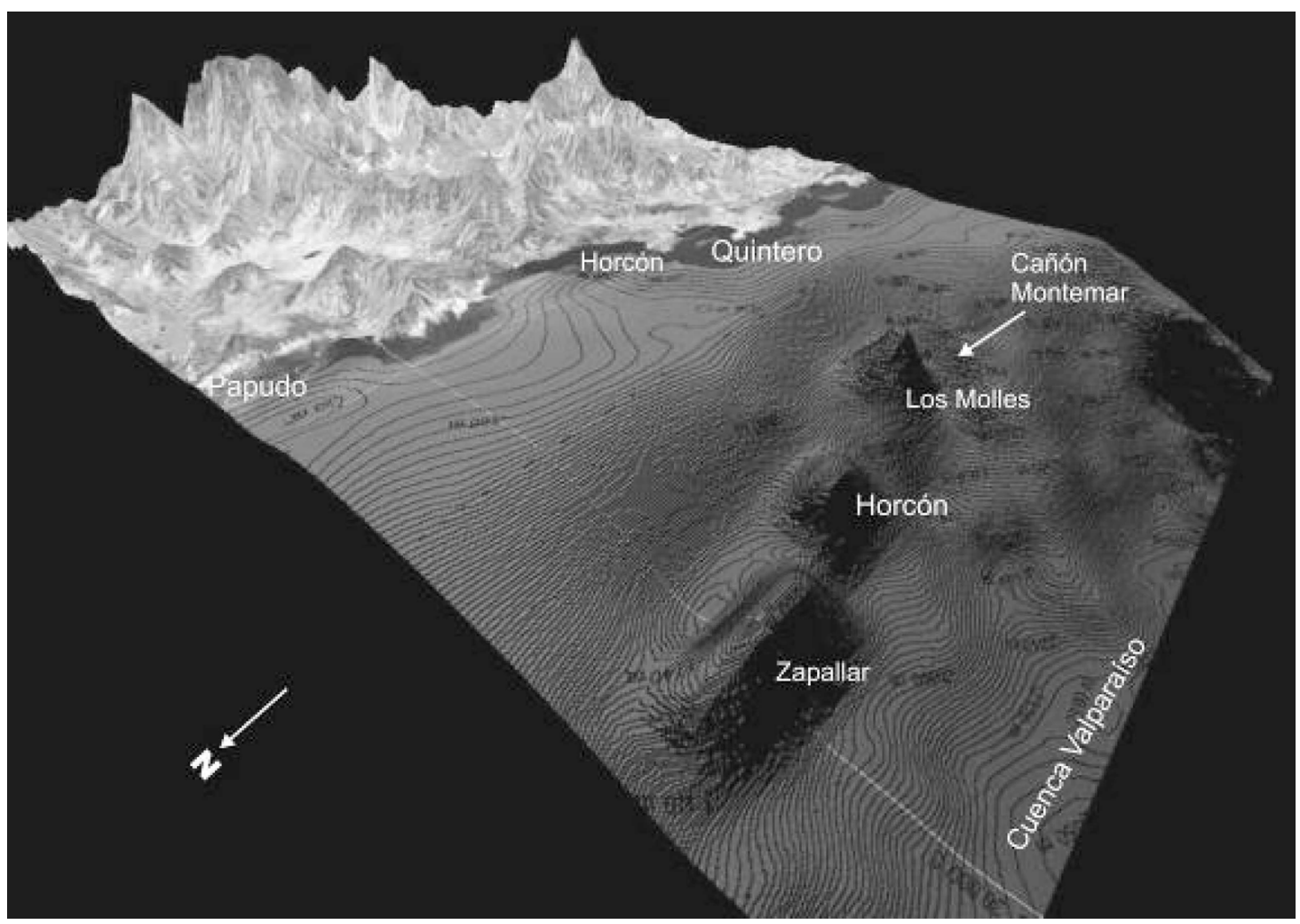

Figura 2

Vista tridimensional del área de la Fig. 1, destacando la morfología en echelon y ubicación de altos estructurales escalonados entre la plataforma y el talud superior

Three-dimensional view of Fig. 1 area, emphasizing the morphology in echelon and location of the structural highs between the shelf and the upper slope 

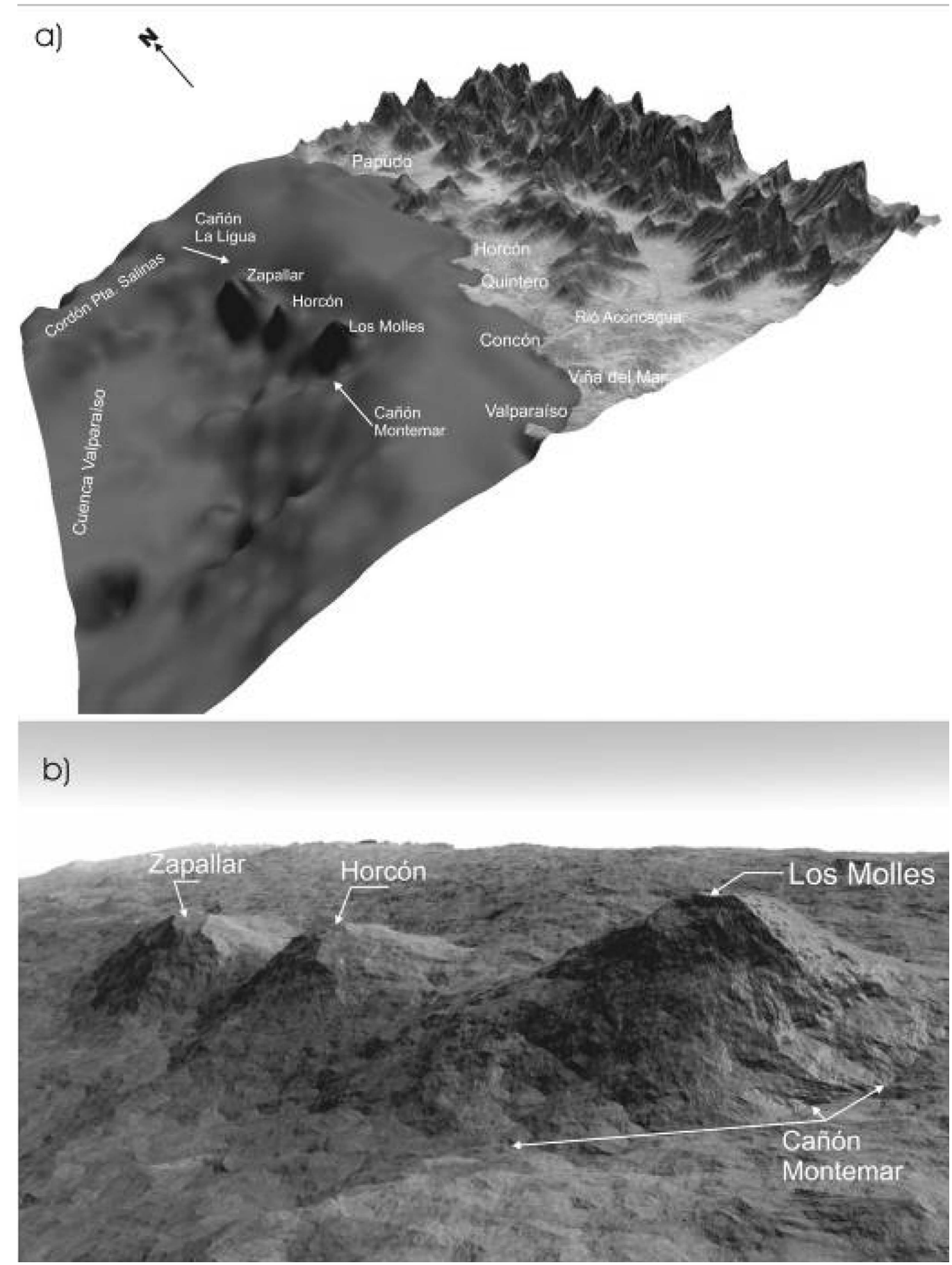

Figura 3

Dos modelos digitales del área. El primero (a), muestra las cumbres limitadas por el cañón La Ligua al norte y Montemar al sur. El segundo (b), es una simulación en realidad virtual de la batimetría que destaca la traza del cañón Montemar

Two digitalized models of the area. The first one (a), shows the highs bounded by La Ligua Canyon in the north, and Montemar Canyon in the south. The second one (b), is a virtual reality simulation of the bathymetry standing out the Montemar tracks 


\section{Tabla 1}

Nombre, posición, profundidad, altura y superficie de los tres altos estructurales

Name, position, depth, height and area of the three structural highs

\begin{tabular}{|c|c|c|c|}
\hline Nombre & Zapallar & Horcón & Los Molles \\
\hline \multirow{2}{*}{ Ubicación } & Lat. $32^{\circ} 30^{\prime} 19.74{ }^{\prime} ' S$ & Lat. $32^{\circ} 36^{\prime} 32.8^{\prime \prime} \mathrm{S}$ & Lat. $32^{\circ} 44^{\prime} 12^{\prime \prime} \mathrm{S}$ \\
\hline & Long. $71^{\circ} 47^{\prime} 01.13^{\prime \prime} \mathrm{W}$ & Long. $71^{\circ} 45^{\prime} 00^{\prime \prime} \mathrm{W}$ & Long. $71^{\circ} 43^{\prime} 21^{\prime \prime} \mathrm{W}$ \\
\hline Profundidad cumbre & 880 mbnm (*) & $733 \mathrm{mbnm}$ & $200 \mathrm{mbnm}$ \\
\hline Área en la base & $111 \mathrm{~km}^{2}$ & $66,9 \mathrm{~km}^{2}$ & $87,8 \mathrm{~km}^{2}$ \\
\hline Perímetro en la base & $42,2 \mathrm{~km}$ & $31,5 \mathrm{~km}$ & $35,5 \mathrm{~km}$ \\
\hline Altura desde base Este & $410 \mathrm{mbnm}$ & $167 \mathrm{mbnm}$ & $460 \mathrm{mbnm}$ \\
\hline Altura desde base Oeste & $920 \mathrm{mbnm}$ & 1067 mbnm & $1600 \mathrm{mbnm}$ \\
\hline Profundidad base Este & $1290 \mathrm{mbnm}$ & $900 \mathrm{mbnm}$ & $660 \mathrm{mbnm}$ \\
\hline Profundidad base Oeste & $1800 \mathrm{mbnm}$ & $1800 \mathrm{mbnm}$ & $1800 \mathrm{mbnm}$ \\
\hline $\begin{array}{l}\text { A la cuadra del lugar } \\
\text { emergido mas cercano }\end{array}$ & $30,1 \mathrm{~km}$ de Punta Pite & $30,1 \mathrm{~km}$ de Punta Peumo & $\begin{array}{l}\text { 20,3 km de Punta Lunes } \\
\text { (Península Horcón) }\end{array}$ \\
\hline
\end{tabular}

$(*)$ metros bajo el nivel del mar

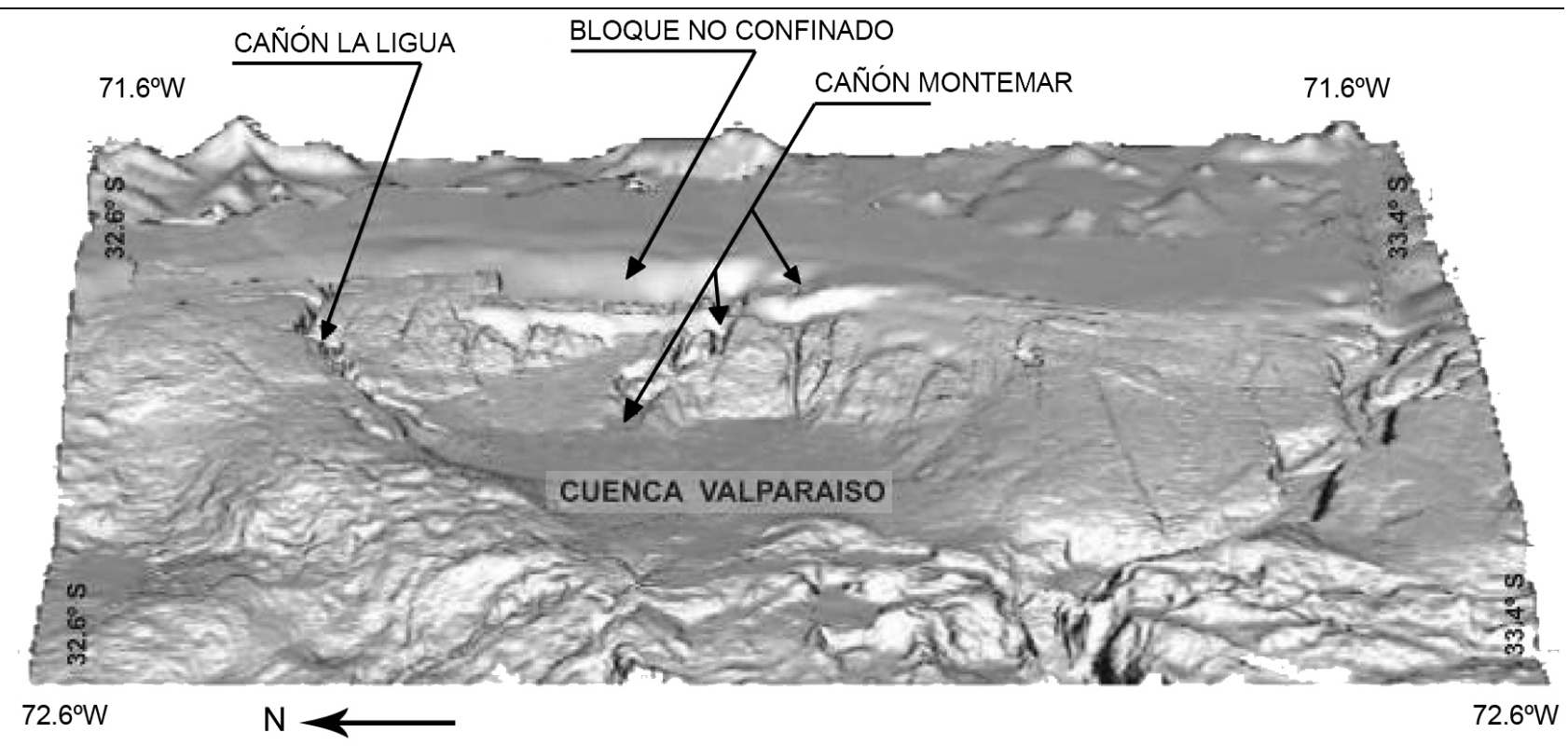

Figura 4

Paisaje regional digitalizado, costa afuera de la región de Valparaíso (modificado de Contreras 2007) ilustrando la subsidencia de bloques no confinados del borde de la plataforma. A esa escala las cumbres aquí descritas no están representados

Regional digitalized landscape offshore of the Valparaiso region (modified from Contreras 2007) showing the outer shelf subsidence of unconfined blocks. At that scale, the highs here described are not represented 
Los antecedentes y los resultados sugieren que con el transcurso del tiempo geológico, el borde de la plataforma continental fue afectado por tensiones causadas - con un alto grado de probabilidad - por esfuerzos de distensión en la parte alta de la corteza continental que implica un cizalle, constituyendo una asimetría escalonada. Como consecuencia de esto, los altos estructurales conforman un bloque colapsado según Contreras (2003). Posteriormente, el mismo autor ilustra esa idea en su tesis de grado (Contreras 2007), lo que se aprecia en la Fig. 4, aunque en ninguna de las dos comunicaciones menciona la morfología de las cumbres que aquí se detallan.

\section{Conclusiones}

Se demuestra la existencia de tres altos estructurales costa afuera de la región de Valparaíso, hasta ahora desconocidos debido a que el área generalmente es deficitaria en información geológica.

Los esfuerzos de tensión y la génesis de las cumbres se asigna al Holoceno superior (últimos 4.000 años AP) como parte de un proceso tectónico ligado a la zona de ruptura caracterizada por enjambres de sismos vecinos a la aspereza Papudo (monte submarino subductado), de la placa descendente, que interactúa con estructuras preexistentes de la placa Sudamericana.

\section{Agradecimientos}

Los autores agradecen profundamente a tres revisores anónimos la dedicación y el tiempo empleado en la crítica de esta investigación; sus observaciones contribuyeron a enriquecerlo significativamente. El primer autor desea extender un póstumo recuerdo y agradecimiento al sismólogo Dr. Lautaro Ponce Mori (QEPD -1996) a quien conoció en marzo de 1985 durante su investigación postsismo en la zona central de Chile. La frase de su informe de que el número anómalo de réplicas de gran magnitud pudieran deberse a la existencia de una pequeña unidad tectónica en la zona de subducción, que pudiera corresponder físicamente a la subducción de altos topográficos, así como más tarde, cuando conversó sobre su temprana hipótesis de un posible quiebre en el margen continental, han sido recordadas desde entonces y parte de ello ha sido desarrollado en este trabajo.

\section{Literatura citada}

Alvarez L. 1964. Geología del área Valparaíso-Viña del Mar. Boletín. Instituto de Investigaciones Geológicas 16: 1-58.

Cecione G. 1980. Darwin's Navidad embayment Santiago Region, Chile as a model of the southeastern Pacific shelf. Journal of Petroleum Geology 2: 309-321.
Contreras S, D Comte, E Vera, PO Thierer, ER Flueh, H Kopp, F Tilmann. 2003. Análisis de la sismicidad registrada por redes terrestres y submarinas: correlación con las principales estructuras batimétricas frente a Valparaíso, Chile central. Resúmenes X Congreso Geológico Chileno, Concepción, 6-10 octubre 2003.

Contreras S. 2007. Análisis de la sismicidad registrada por redes terrestres y submarinas: correlación con las principales estructuras batimétricas frente a Valparaíso, Chile Central, e implicancias medio ambientales derivadas de la ocurrencia de hidratos de gas en el margen continental. Tesis de Magíster, Departamento de Geofísica, Facultad de Ciencias Físicas y Matemáticas, Universidad de Chile, Santiago, 112 pp.

Frassinetti D \& V Covacevich.1993. Bivalvos del Mioceno marino de Matanzas (Formación Navidad, Chile central). Boletín del Museo Nacional de Historia Natural 44: 7397.

Intemational Hydrographic Bureau. 2001. Standardization of Undersea Feature Names: guidelines, proposal form, terminology. Bathymetric Publication 6: 1-50.

Latorre C, PI Moreno, G Vargas, A Maldonado, R VillaMartínez, JJ Armesto, C Villagrán, M Pino, L Núñez \& M Grosjean. 2007. Late Quaternary environments and paleoclimate. En: Moreno T \& W Gibbons (eds), The Geology of Chile, pp. 309-328. London Geological Society Press, London.

Laursen J \& WR Normark. 2002. Late Quaternary evolution of the San Antonio submarine canyon in the central Chile forearc $\left(\sim 33^{\circ} \mathrm{S}\right)$. Marine Geology 188: 365-390.

Martínez-Pardo R. 1990. Major Neogene events of Southeastern Pacific: the Chilean and Peruvian record. Palaeogeography, Palaeoclimatology, Palaeoecology 77: 263-278.

Sandwell DT \& WHF Smith. 1997. Marine gravity anomaly from Geosat and ERS-1 satellite altimetry. Journal of Geophysical Research 102: 10.039-10.054.

Sandwell DT \& WHF Smith. 2001. Bathymetric estimation. En: L-L Fu \& A Cazenave (editors). Satellite Altimetry and Earth Sciences, International Geophysical Series 69: 441457, Academic Press, New York.

Sandwell DT, WHF Smith, S Gille, E Kappel, S Jayne, K Soofi, B Coakley \& L Géli. 2006. Bathymetry from space: Rationale and requirements for a new, high-resolution altimetric mission. Comples Rendus Geoscience 338:10491062 , Issue 14/15.

Scholl D, MN Christensen, R von Huene \& M Marlow. 1970. Peru-Chile Trench sediment and sea-floor spreading. Bulletin of the Geological Society of America 81: 13391360.

Stoffers P \& Shipboard Scientific Party. 1992. Cruise report Sonne 80a - Midplate oceanic volcanism in the Southeast Pacific Berichte, 128 pp. Universität Kiel, Kiel. 
Tapley B \& K Kim. 2001. Applications to Geodesy. En: L-L $\mathrm{Fu} \&$ A Cazenave (eds). Satellite Altimetry and Earth Sciences, International Geophysical Series 69: 371-403, Academic Press, Nueva York.

Tavera J. 1979. Estratigrafía y paleontología de la Formación Navidad, Provincia de Colchagua, Chile (Lat $30^{\circ} 50^{\prime}-34^{\circ}$ S). Boletín del Museo Nacional de Historia Natural 36: 1176.

Thierer P, ER Flueh, H Kopp, F Tilmann, D Comte \& S Contreras. 2005. Local earthquake monitoring offshore Valparaiso, Chile, pp. J73-J83. En: Miller R (ed). Contributions to Latin-American Geology, $18^{\text {th }}$ LatínAmerican Colloquium, Freiburg, 2003, pp. 1-265.

Valenzuela E, H Vergara \& E Reyes. 1979. Petrología sedimentaria del litoral y la plataforma continental de

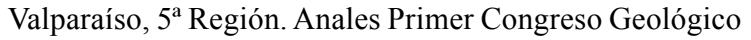
Chileno, Arica 3: I-101-I-121.
Vergara H. 1996. La cuenca de Valparaíso: antecedentes morfotectónicos y sedimentológicos. Revista de Biología Marina 31(1): 45-63.

von Huene R, J Corvalán, ER Flueh, K Hinz, J Korstgard, CR Ranero, W Weinrebe \& the CONDOR Scientist. 1997. Tectonic control of the subducting Juan Fernandez Ridge on the Andean margin near Valparaíso, Chile. Tectonics 16(3): 474-488.

Yáñez G, CR Ranero, R von Huene \& J Díaz. 2001. Magnetic anomaly interpretation across the southern central Andes $\left(32^{\circ}-34^{\circ} \mathrm{S}\right)$ : The role of the Juan Fernandez Ridge in the late Tertiary evolution of the margin. Joumal of Geophysical Research 106(B4): 6325-6345.

Recibido el 28 de abril de 2008 y aceptado el 25 de septiembre de 2008 